\title{
OSCILLATIONS OF THE MAGNETIC NETWORK
}

\author{
BRUCE W. LITES
}

High Altitude Observatory, NCAR, P.O. Box 3000, Boulder CO 80307 . 3000 , USA

\author{
ROBERT J. RUTTEN \\ Sterrekundig Instituut, Postbus 80000 , NL-3508 TA Utrecht, The Nether- \\ lands \\ WOLFGANG KALKOFEN \\ Harvard-Smithsonian Center for Astrophysics, 60 Garden Street, Cam- \\ bridge MA 02138, USA
}

\begin{abstract}
We present high-quality power spectra of oscillations in the quiet solar chromosphere measured from the $\mathrm{Ca} I \mathrm{H}$ line. They show unambiguously that the network does not participate in chromospheric three-minute oscillations, but that instead it oscillates in slow wave motions which are neither present in the surrounding non-magnetic chromosphere, nor in the underlying photosphere.
\end{abstract}

\section{INTRODUCTION}

Chromospheric oscillations are of great interest in the context of cool-star chromospheric heating. The small-scale dynamics of the solar chromosphere supply the primary testing ground to constrain and identify heating mechanisms, whether magnetic or acoustic in origin ( $c f$. Ulmschneider et al. 1991). Proper diagnostics are required, however; in particular, vertical discrimination is required between photospheric and chromospheric layers, and horizontal discrimination between the network and internetwork regimes.

Separation between network and internetwork has been made in oscillation analyses employing the $\mathrm{Ca}$ II infrared lines (Lites et al. 1982, Deubner \& Fleck 1990) and $\mathrm{H} \alpha$ (von Uexküll et al. 1989) but these lines offer limited height discrimination. The source functions of the $\mathrm{Ca}$ II infrared lines are controlled by $\mathrm{H} \& \mathrm{~K}$ scattering (Uitenbroek 1989) and respond to changes over a wide height range (Mein 1971). H $\alpha$ has a doubly-peaked contribution function (Schoolman 1972).

$\mathrm{Ca}$ II H \& $\mathrm{K}$ offer the cleanest chromospheric diagnostics accessible from the ground. The principal Fourier study of their oscillations is by Cram (1978). He did not distinguish between network and internetwork. We improve on his work by measuring oscillations in the core of $\mathrm{Ca}$ II $\mathrm{H}$ with such discrimination. 


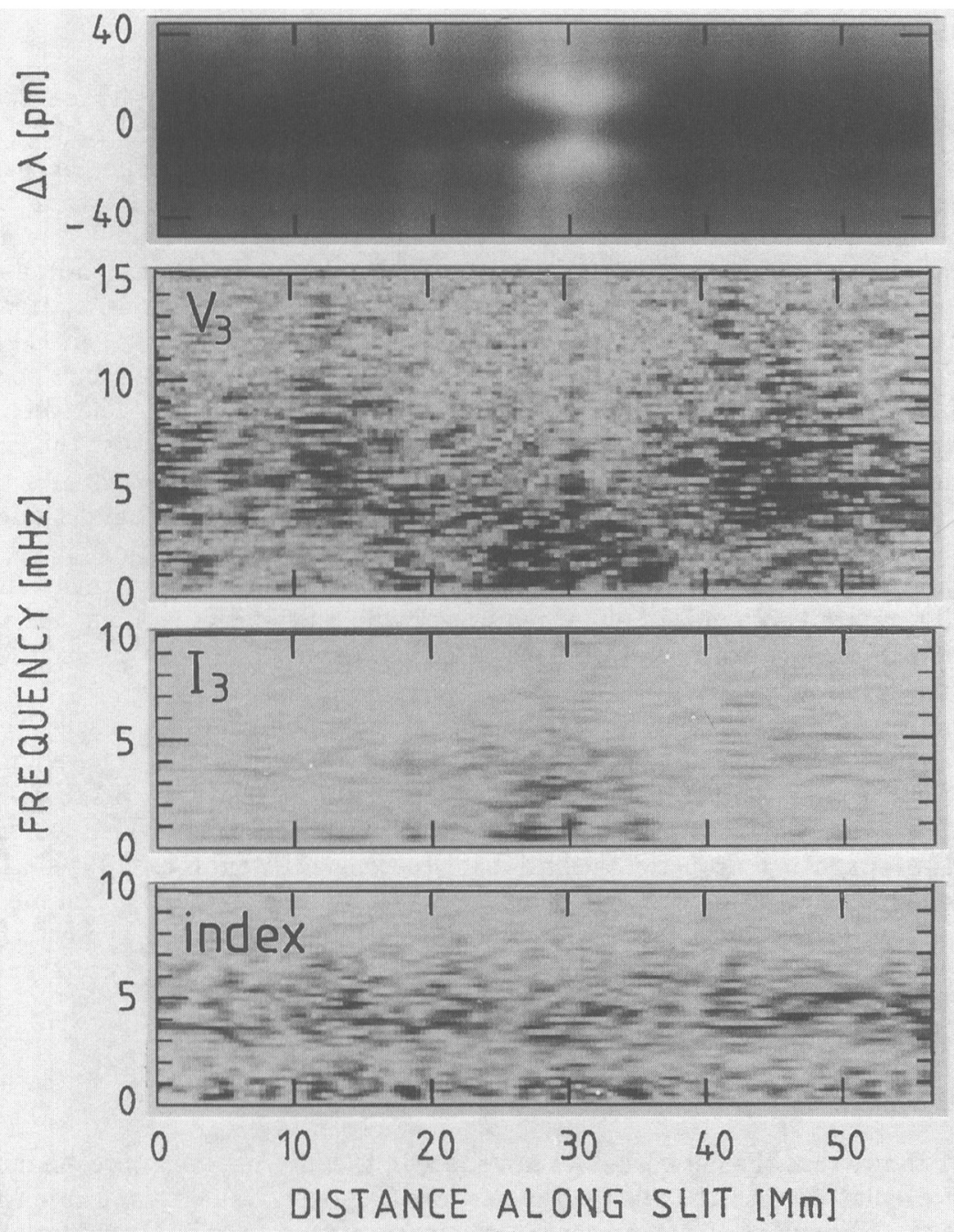

FIGURE I Spatially resolved chromospheric oscillations in CaII H. Grey scales measure monochromatic intensity (top, bright is large) and Fourier power (other panels, dark is large). Abscissae: spatial dimension along spectrograph slit. Top: time-averaged intensity profiles. Second panel: power in line-center Doppler variations $\left(V_{3}\right)$. Third panel: power in line-center intensity variations $\left(I_{3}\right)$. Bottom: power in integrated-intensity variations ( $\mathrm{H}$-index; width $\Delta \lambda=90 \mathrm{pm}$ ).

The network areas crossed by the slit are marked by bright $\mathrm{H}_{2 V} \& \mathrm{H}_{2 R}$ emission in the top panel. Their line-center oscillation characteristics differ strikingly from the internetwork three-minute $(f=3-10 \mathrm{mHz})$ oscillations seen in $V_{3}$. Both $V_{3}$ and $I_{3}$ show large low-frequency power $(f<3 \mathrm{mHz})$ in the network areas. The $\mathrm{H}$-index panel contains bands of enhanced power for $f<2 \mathrm{mHz}$ and $f=3-7 \mathrm{mHz}$, both without obvious network/internetwork distinction. 


\section{OBSERVATIONS}

Our data consist of a one-hour sequence of $\mathrm{CaII} \mathrm{H}$ spectrograms taken at $5 \mathrm{~s}$ cadence with the NSO/SP Vacuum Tower Telescope in 1984. Figure 1 shows spectra that are spatially resolved along the spectrograph slit. The top panel displays time-averaged intensity spectra and shows bright network $\mathrm{H}_{2 V}$ and $\mathrm{H}_{2 R}$ features around $x=30 \mathrm{Mm}$ and weaker emission near $x=20 \mathrm{Mm}$. These network locations stand out very clearly in the $\mathrm{H}_{3}$ Dopplershift $\left(V_{3}\right.$, second panel) and intensity $\left(I_{3}\right.$, third panel) power spectra. The three-minute oscillation is prominently present only within the internetwork regions, and only in the $V_{3}$ panel. In stark contrast, the network areas crossed by the slit have much power in both $V_{3}$ and $I_{3}$ only at low frequencies, below $f=5 \mathrm{mHz}$. The vertical column at $x=30 \mathrm{Mm}$ betrays seeing jitter of a small structure across the slit.

The bottom panel ( $\mathrm{H}$-index) shows intensity power in a $\Delta \lambda=0.09 \mathrm{~nm}$ wide band around line center. This index is representative of narrow-band $\mathrm{CaII} \mathrm{K}$ filtergrams and of the Mt. Wilson $\mathrm{H} \& \mathrm{~K}$ photometer. The addition of innerwing photons produces strikingly different characteristics compared with the $I_{3}$ panel. There are two bands of enhanced power which extend fully along the slit; the presence of network is not obvious in either band. Only the $x=30 \mathrm{Mm}$ network column is weakly present.

The upper band in the $\mathrm{H}$-index panel extends to higher frequencies than the acoustic oscillations of the photosphere (which cover the $f=2.5-5 \mathrm{mHz}$ range). It contains the three-minute "whiskers" seen in the inner H \& K wings in spatially resolved spectrograms (Beckers \& Artzner 1974), which portray upwards-propagating acoustic oscillations (Rutten \& Uitenbroek 1991). The low-frequency $(f<2 \mathrm{mHz}$ ) band of power was observed earlier by Schmieder (1976), Cram (1978) and Deubner \& Fleck (1989) and attributed by these authors to gravity waves. It is not present in the $\mathrm{Ca}$ II $\mathrm{H}$ line-center power spectra ( $V_{3}$ and $I_{3}$ panels).

\section{DISCUSSION}

Figure 1 shows that the network does not share in the internetwork three-minute velocity oscillation, but that instead it has low-frequency velocity and intensity power of its own which is not present in the internetwork, nor in the underlying photosphere (the latter follows from additional data, presented in a longer paper which we have submitted to ApJ).

Such low-frequency network power has been observed before, but far less cleanly and clearly than here. Damé et al. (1984) noted enhanced power for network areas in their $0.12 \mathrm{~nm}$ FHWM K-line filtergrams. Our bottom panel shows that such wide-band observations $\mathrm{mix}$ in too much lower-atmosphere signal from the CaII wings to obtain a clear signature of the $I_{3}$ network power.

The Ca II infrared line analyses of Lites et al. (1982) and Deubner \& Fleck (1990) suffer similarly from lower height of formation and from lack of height discrimination compared with the $\mathrm{H} \& \mathrm{~K}$ cores. For example, all power spectra of Deubner \& Fleck (1990) display prominent five-minute peaks and dips at $2-3 \mathrm{mHz}$ that are seen here only in the $\mathrm{H}$-index panel. This confirms that the $\mathrm{Ca}$ II infrared lines correspond primarily to the inner wings of $\mathrm{H} \& \mathrm{~K}$ in formation 
(Rutten \& Uitenbroek 1991), with mixed-in chromospheric sensitivity through coupling to the $\mathrm{H} \& \mathrm{~K}$ cores with NLTE-enhanced response (Uitenbroek 1989).

The H $\alpha$ observations of von Uexküll et al. (1989) come closest to these results, especially their Fig. $3 \mathrm{e}-\mathrm{f}$ in which the brightest, respectively darkest pixels were used to obtain network and internetwork velocity power spectra. However, their intensity power spectra contain a $f=1 \mathrm{mHz}$ peak for both network and internetwork which corresponds to the low-frequency band in our $\mathrm{H}$-index panel. It is absent in the $I_{3}$ and $V_{3}$ panels. Thus, the $\mathrm{H} \alpha$ intensity seems sensitive to rather low-lying layers corresponding to $\mathrm{H} \& \mathrm{~K}$ inner-wing formation.

What causes the low-frequency network power in $V_{3}$ and $I_{3}$ ? Von Uexküll et al. argue that it portrays stochastic motions. We believe from time-resolved displays and from new, similar data that these motions are oscillatory in nature. We suspect that these slow undulations represent a key phenomenon to identify the nature of chromospheric energy dissipation in magnetic elements. Obviously - to quote our Colloquium Chairman -we need more data.

\section{REFERENCES}

Beckers, J. M., Artzner, G. 1974, Solar Phys., 37, 309

Cram, L. E. 1978, A\&A, 70, 345

Damé, L., Gouttebroze, P., Malherbe, J.-M. 1984, A\&A, 130, 331

Deubner, F.-L., Fleck, B. 1989, A\&A, 213, 423

Deubner, F.-L., Fleck, B. 1990, A\&A, 228, 506

Lites, B. W., Chipman, E. G., White, O. R. 1982, ApJ, 253, 367

Mein, P. 1971, Solar Phys., 20, 3

Rutten, R. J., Uitenbroek, H. 1991, Solar Phys., 134, 15

Schmieder, B. 1976, Solar Phys., 47, 435

Schoolman, S. A. 1972, Solar Phys., 22, 344

Uitenbroek, H. 1989, A\&A, 213, 360

Ulmschneider, P., Priest, E. R., Rosner, R. (eds.) 1991, Mechanisms of Chromospheric and Coronal Heating, Proc. Int. Heidelberg Conference, SpringerVerlag, Berlin

von Uexküll, M., Kneer, F., Malherbe, J. M., Mein, P. 1989, A\&A, 208, 290 